\title{
STRONGLY SINGULAR INTEGRAL OPERATORS ASSOCIATED TO DIFFERENT QUASI-NORMS ON THE HEISENBERG GROUP
}

\author{
Norberto Laghi AND NeIL Lyall
}

\begin{abstract}
In this article we study the behavior of strongly singular integrals associated to three different, albeit equivalent, quasi-norms on Heisenberg groups; these quasinorms give rise to phase functions whose mixed Hessians may or may not drop rank along suitable varieties. In the particular case of the Koranyi norm we improve on the arguments in [7] and obtain sharp $L^{2}$ estimates for the associated operators.
\end{abstract}

\section{Introduction}

The Heisenberg group $\mathbf{H}_{a}^{n}$ is a non-commutative nilpotent Lie group, with underlying manifold $\mathbf{R}^{2 n+1}$ equipped the group law

$$
(x, t) \cdot(y, s)=\left(x+y, s+t-2 a x^{\mathrm{t}} J y\right)
$$

where $a$ is a nonzero real number and $J$ denotes the standard symplectic matrix on $\mathbf{R}^{2 n}$, namely

$$
J=\left(\begin{array}{cc}
0 & I_{n} \\
-I_{n} & 0
\end{array}\right)
$$

with inverses given by $(x, t)^{-1}=-(x, t)$. The nonisotropic dilations

$$
(x, t) \mapsto\left(\delta x, \delta^{2} t\right) .
$$

are automorphisms of $\mathbf{H}_{a}^{n}$ and as such the homogeneous dimension of this group is $2 n+2$.

We will consider here, for different quasi-norms $\rho(x, t)$ on $\mathbf{H}_{a}^{n}$, the class of model (group) convolution operators formally given by

$$
T f(x, t)=f * K_{\alpha, \beta}(x, t)
$$

where $K_{\alpha, \beta}$ is a strongly singular distributional kernel on $\mathbf{H}_{a}^{n}$ that agrees, for $(x, t) \neq$ $(0,0)$, with the function

$$
K_{\alpha, \beta}(x, t)=\rho(x, t)^{-2 n-2-\alpha} e^{i \rho(x, t)^{-\beta}} \chi(\rho(x, t)),
$$

where $\beta>0$ and $\chi$ is smooth and compactly supported in a small neighborhood of the origin.

Operators of this type were first studied, using Fourier transform techniques, in the Euclidean setting of $\mathbf{R}^{d}$ with $\rho(x)=|x|$ by Hirschman [4] in the case $d=1$ and then

Received by the editors July 31, 2006.

2000 Mathematics Subject Classification. 42B20, 43A80.

Key words and phrases. Strongly singular integrals, Heisenberg group.

Both authors were partially supported by HARP grants from the European Commission; the first author is currently supported by an EPSRC grant, the second author is partially supported by a NSF FRG grant. 
in higher dimensions by Wainger [10], C. Fefferman [2], and C. Fefferman and Stein [3]. For some generalizations and an oscillatory integral approach to these classical results, see Lyall [6]. The analogous questions on the Heisenberg group were first investigated by the second author in [7].

1.1. Statement of main results. There are various natural choices of quasi-norm on $\mathbf{H}_{a}^{n}$, for example one can take

$$
\rho_{0}(x, t)=\max \left\{\left|x_{1}\right|, \ldots,\left|x_{2 n}\right|,|t|^{1 / 2}\right\},
$$

however this choice is not smooth away from the origin. We shall instead consider the following three equivalent quasi-norms which clearly are smooth away from the origin;

(i) $\rho_{1}(x, t)=\left(|x|^{4}+t^{2}\right)^{1 / 4}$

(ii) $\rho_{2}$ defined by $\rho_{2}(x, t)=1 \Longleftrightarrow|x|^{2}+t^{2}=1$ and extended by homogeneity

(iii) $\rho_{3}(x, t)=\left(x_{1}^{4}+\cdots+x_{2 n}^{4}+t^{2}\right)^{1 / 4}$.

The case when $\rho(x, t)=\rho_{1}(x, t)$, the so called Koranyi norm on the Heisenberg group, was initially studied by the second author in [7] using the group Fourier transform.

Theorem (Lyall [7]). Let $\rho(x, t)=\rho_{1}(x, t)$ and $a \neq 0$.

(i) If $\alpha \leq n \beta$, then $T$ extends to a bounded operator from $L^{2}\left(\mathbf{H}_{a}^{n}\right)$ to itself.

(ii) If $T$ extends to a bounded operator from $L^{2}\left(\mathbf{H}_{a}^{n}\right)$ to itself, then $\alpha \leq\left(n+\frac{1}{2}\right) \beta$.

The proof of this result relied upon the radial nature of the Koranyi norm and uniform asymptotic expansions for Laguerre functions due to Erdélyi [1] along with some careful analysis.

In this article we optimally sharpen the previously obtained estimates and in addition address the question of the behavior of different quasi-norms for the first time. We do not employ the Fourier transform in our arguments and as such our methods are not restricted to the class of translation-invariant operators.

We fix the constant

$$
C_{\beta}=\frac{\beta+2}{2}\left(2 \beta+5+\sqrt{(2 \beta+5)^{2}-9}\right)
$$

and note that $C_{\beta} \geq 9$ for all $\beta>0$. Our first main result is then the following.

Theorem 1. If $\rho(x, t)=\rho_{1}(b x, b t)$ with $0<a^{2} / b^{2}<C_{\beta}$, then $T$ extends to a bounded operator from $L^{2}\left(\mathbf{H}_{a}^{n}\right)$ to itself if and only if $\alpha \leq\left(n+\frac{1}{2}\right) \beta$.

The analogous result for $\rho(x, t)=\rho_{2}(x, t)$, the nonisotropic Minkowski functional associated to the (Euclidean) unit ball, is the following.

Theorem 2. If $\rho(x, t)=\rho_{2}(b x, b t)$ with $a^{2} / b^{2} \leq 1$, then $T$ extends to a bounded operator from $L^{2}\left(\mathbf{H}_{a}^{n}\right)$ to itself whenever $\alpha \leq\left(n+\frac{1}{2}\right) \beta$.

We note that the Koranyi norm $\rho_{1}(x, t)$ is indeed an actual norm on $\mathbf{H}_{a}^{n}$ for all $0<a^{2} \leq 1$, as is the nonisotropic Minkowski functional $\rho_{2}(x, t)$ for sufficiently small $|a|$.

A negative result for the quasi-norm $\rho_{3}(x, t)$ that relates to the proofs of Theorems 1 and 2 (the direct relationship is with Proposition 7) is discussed in Section 5. 


\section{Reductions and remarks}

2.1. Reduction to dyadic estimates. The necessary condition in Theorem 1 follows from the arguments in [7]. To establish sufficiency in both Theorems 1 and 2 matters reduce to considering the dyadic operators

$$
T_{j}(x, t)=f * K_{j}(x, t),
$$

where

$$
K_{j}(x, t)=\vartheta\left(2^{j} \rho(x, t)\right) K_{\alpha, \beta}(x, t),
$$

with $\vartheta \in C_{0}^{\infty}(\mathbf{R})$ supported in $\left[\frac{1}{2}, 2\right]$ is chosen such that $\sum_{j=0}^{\infty} \vartheta\left(2^{j} r\right)=1$ for all $0 \leq r \leq 1$.

As in [7] everything reduces to establishing the following key dyadic estimates.

Theorem 3. If $\alpha \leq\left(n+\frac{1}{2}\right) \beta$ and either

(i) $\rho(x, t)=\rho_{1}(b x, b t)$ with $0<a^{2} / b^{2}<C_{\beta}$, or

(ii) $\rho(x, t)=\rho_{2}(b x, b t)$ with $a^{2} / b^{2} \leq 1$,

then the dyadic operators $T_{j}$ are bounded uniformly on $L^{2}\left(\mathbf{H}_{a}^{n}\right)$, more precisely

$$
\int_{\mathbf{H}_{a}^{n}}\left|T_{j} f(x, t)\right|^{2} d x d t \leq C 2^{j(2 \alpha-(2 n+1) \beta)} \int_{\mathbf{H}_{a}^{n}}|f(x, t)|^{2} d x d t .
$$

Theorems 1 and 2 then follow from an application of Cotlar's lemma (and a standard limiting argument) since the operators $T_{j}$ are, in the following sense, almost orthogonal.

Proposition 4. If $\alpha \leq\left(n+\frac{1}{2}\right) \beta$, then

$$
\left\|T_{j}^{*} T_{j^{\prime}}\right\|_{L^{2}\left(\mathbf{H}_{a}^{n}\right) \rightarrow L^{2}\left(\mathbf{H}_{a}^{n}\right)}+\left\|T_{j} T_{j^{\prime}}^{*}\right\|_{L^{2}\left(\mathbf{H}_{a}^{n}\right) \rightarrow L^{2}\left(\mathbf{H}_{a}^{n}\right)} \leq C 2^{-\left(n+\frac{1}{2}\right) \beta\left|j-j^{\prime}\right|} .
$$

This follows exactly as in [7] once we have made the observation that if $\rho(x, t)$ is any quasi-norm on $\mathbf{H}_{a}^{n}$ satisfying the estimate $c^{-1} \leq \rho\left(\delta x, \delta^{2} t\right) \leq c$ for some $c \geq 1$ and $\delta>0$, then there exists a constant $c_{0}>1$ so that either

$$
c_{0}^{-1} \leq \frac{\partial}{\partial x_{j}} \rho(x, t) \leq c_{0}
$$

for some $j=1, \ldots, 2 n$, or

$$
c_{0}^{-1} \delta \leq \frac{\partial}{\partial t} \rho(x, t) \leq c_{0} \delta .
$$

Since from this it follows that

$$
\left|\nabla_{(y, s)}\left[\rho(y, s)^{-\beta}-\rho((x, t) \cdot(y, s))^{-\beta}\right]\right| \geq C>0,
$$

whenever $\rho((x, t) \cdot(y, s)) \gg \rho(y, s)$. For more details see [7]. 
2.2. Remarks on $L^{p} \rightarrow L^{q}$ estimates. For $p \leq q$, the $L^{p} \rightarrow L^{q}$ operator norms of $T_{j}$ are equal to that of the rescaled operator $\widetilde{T}_{j}$ given by

$$
\widetilde{T}_{j} f(x, t)=2^{j h(1 / p-1 / q)} 2^{j \alpha} S_{j} f(x, t)
$$

where $h=2 n+2$ denotes the homogeneous dimension of $\mathbf{H}_{a}^{n}$ and

$$
S_{j} f(x, t)=\int_{\mathbf{H}_{a}^{n}} \widetilde{K}_{j}\left((y, s)^{-1} \cdot(x, t)\right) f(y, s) d y d s
$$

with

$$
\widetilde{K}_{j}(x, t)=\vartheta(\rho(x, t)) \rho(x, t)^{-h-\alpha} e^{i 2^{j \beta} \rho(x, t)^{-\beta}} .
$$

In particular establishing Theorem 3 is equivalent to showing that the $L^{2}$ operator norm of $S_{j}$ is $O\left(2^{-j d \beta / 2}\right)$, where $d=2 n+1$ denotes the topological dimension of $\mathbf{H}_{a}^{n}$, standard interpolation techniques then give that the $L^{p}$ operator norm of $S_{j}$ is $O\left(2^{-j d \beta / 2} 2^{j d \beta|1 / p-1 / 2|}\right)$. If $p \leq 2$ this is also a bound for the $L^{p} \rightarrow L^{p^{\prime}}, L^{p} \rightarrow L^{2}$, and $L^{p^{\prime}} \rightarrow L^{2}$ operator norms and can, for this range of $p$, be written more succinctly as $O\left(2^{-j d \beta / p^{\prime}}\right)$.

From the corresponding results for the dyadic operator $T_{j}$, which of course follow immediately from those for $S_{j}$, we obtain the following.

Theorem 5. If $1<p<\infty$ and either

(i) $\rho(x, t)=\rho_{1}(b x, b t)$ with $0<a^{2} / b^{2}<C_{\beta}$, or

(ii) $\rho(x, t)=\rho_{2}(b x, b t)$ with $a^{2} / b^{2} \leq 1$,

then $T$ extends to a bounded operator from $L^{p}\left(\mathbf{H}_{a}^{n}\right)$ to itself whenever

$$
\left|\frac{1}{p}-\frac{1}{2}\right|<\frac{d \beta-2 \alpha}{2 d \beta}
$$

The estimates above match those obtained by Wainger [10] for the corresponding operators in $\mathbf{R}^{d}$. However, unlike the situation in the Euclidean setting which was settled in [2] and [3], the behavior of the operator $T$ on the Heisenberg group near $L^{1}$ and the endpoint results in $L^{p}$ for $p \neq 2$ remain open problems.

We chose to use the notation $h$ and $d$ for the homogeneous and topological dimensions of $\mathbf{H}_{a}^{n}$ in the arguments alluded to above as these also apply in the setting of homogeneous groups; of course establishing the analogue of Theorem 3 in this more general group settings remains an open problem.

\section{Homogeneous groups and a proposition of Hörmander}

The Heisenberg group is of course one of the simplest examples of a (non-commutative) homogeneous group. Recall that a homogeneous group consists of $\mathbf{R}^{d}$ equipped with a Lie group structure, together with a family of dilations

$$
x=\left(x_{1}, \ldots, x_{d}\right) \mapsto \delta \circ x=\left(\delta^{a_{1}} x_{1}, \ldots, \delta^{a_{d}} x_{d}\right),
$$

with $a_{1}, \ldots, a_{d}$ strictly positive, that are group automorphisms, for all $\delta>0$.

To each homogeneous group on $\mathbf{R}^{d}$, we can associate its Lie algebra, consisting of left-invariant vector fields on $\mathbf{R}^{d}$, with basis $\left\{X_{j}\right\}_{1 \leq j \leq d}$ where each $X_{j}$ is the leftinvariant vector field that agrees with $\partial / \partial x_{j}$ at the origin.

Key to establishing Theorem 3 is the following, presumably well known, generalization of a proposition of Hörmander [5], see also [9], Chapter IX. 
Proposition 6. Let $\Psi$ be a smooth function of compact support in $x$ and $y$, and $\Phi$ be real-valued and smooth on the support of $\Psi$. If we assume that

$$
\operatorname{det}\left(X_{j} Y_{k} \Phi(x, y)\right) \neq 0
$$

on the support of $\Psi$, then for $\lambda>0$ we have

$$
\left\|\int_{\mathbf{R}^{d}} \Psi(x, y) e^{i \lambda \Phi(x, y)} f(y) d y\right\|_{L^{2}\left(\mathbf{R}^{d}\right)} \leq C \lambda^{-\frac{d}{2}}\|f\|_{L^{2}\left(\mathbf{R}^{d}\right)} .
$$

Proposition 6 can in fact be extended to families of smooth vector fields $X_{1}, \ldots, X_{d}$ forming a basis at every point of $\mathbf{R}^{d}$; however, we choose to state it in this restricted generality (which is already more than we need) as this admits a proof which is simply the natural modification of Hörmander's original argument.

Proof. By using a partition of unity we may assume that the amplitude $\Psi$ has suitably small compact support in both $x$ and $y$. Denoting the operator on the left hand side of inequality (5) by $T_{\lambda}$ it is then easy to see that

$$
T_{\lambda}^{*} T_{\lambda} f(y)=\int_{\mathbf{R}^{d}} K_{\lambda}(x, z) f(z) d z
$$

where

$$
K_{\lambda}(x, z)=\int_{\mathbf{R}^{d}} e^{i \lambda[\Phi(x, y)-\Phi(z, y)]} \Psi(x, y) \overline{\Psi(z, y)} d y .
$$

It therefore suffices to establish the kernel estimate

$$
\left|K_{\lambda}(x, z)\right| \leq C\left(1+\lambda\left|z^{-1} \cdot x\right|\right)^{-N},
$$

since from this it would follow that

$$
\int\left|K_{\lambda}(x, z)\right| d z \approx\left|\left\{z:\left|z^{-1} \cdot x\right| \leq \lambda^{-1}\right\}\right|=C \lambda^{-d}
$$

and similarly for $\int\left|K_{\lambda}(x, z)\right| d x$, and therefore by Schur's test that

$$
\left\|T_{\lambda}^{*} T_{\lambda} f\right\|_{L^{2}\left(\mathbf{R}^{d}\right)} \leq C \lambda^{-d}\|f\|_{L^{2}\left(\mathbf{R}^{d}\right)} .
$$

The kernel $K_{\lambda}(x, z)$ is of course always bounded, hence in order to establish (6) we need only consider the case when $\left|z^{-1} \cdot x\right| \geq \lambda^{-1}$. Now

$$
\begin{aligned}
Y_{k} \Phi(x, y)-Y_{k} \Phi(z, y) & =\int_{0}^{1} \frac{d}{d t} Y_{k} \Phi\left(z \cdot t\left(z^{-1} \cdot x\right), y\right) d t \\
& =\sum_{j=1}^{d}\left(z^{-1} \cdot x\right)_{j} \int_{0}^{1} X_{j} Y_{k} \Phi\left(z \cdot t\left(z^{-1} \cdot x\right), y\right) d t \\
& =\sum_{j=1}^{d}\left(z^{-1} \cdot x\right)_{j} X_{j} Y_{k} \Phi(x, y)+O\left(\left|z^{-1} \cdot x\right|^{2}\right) .
\end{aligned}
$$

So if we let

$$
A=A(x, y)=X_{j} Y_{k} \Phi(x, y) \quad \text { and } \quad u=u(x, y, z)=A^{-1} \frac{z^{-1} \cdot x}{\left|z^{-1} \cdot x\right|}
$$

and define

$$
\Delta(x, y, z)=\left(u_{1} Y_{1}+\cdots+u_{d} Y_{d}\right)[\Phi(x, y)-\Phi(z, y)]
$$


it follows that

$$
\Delta(x, y, z)=\left|z^{-1} \cdot x\right|+O\left(\left|z^{-1} \cdot x\right|^{2}\right) .
$$

Therefore for $\left|z^{-1} \cdot x\right|$ small enough, it is here that we use our initial suitably small support assumption, we have

$$
|\Delta(x, y, z)| \geq \frac{1}{2}\left|z^{-1} \cdot x\right|
$$

and if we now set

$$
D=\frac{1}{i \lambda \Delta(x, y, z)}\left(u_{1} Y_{1}+\cdots+u_{d} Y_{d}\right)
$$

it follows that

$$
\begin{aligned}
& \left|\int_{\mathbf{R}^{d}} e^{i \lambda[\Phi(x, y)-\Phi(z, y)]} \Psi(x, y) \overline{\Psi(z, y)} d y\right| \\
= & \left|\int_{\mathbf{R}^{d}} D^{N}\left(e^{i \lambda[\Phi(x, y)-\Phi(z, y)]}\right) \Psi(x, y) \overline{\Psi(z, y)} d y\right| \\
= & \left|\int_{\mathbf{R}^{d}} e^{i \lambda[\Phi(x, y)-\Phi(z, y)]}\left(D^{\mathrm{t}}\right)^{N}(\Psi(x, y) \overline{\Psi(z, y)}) d y\right| \\
\leq & C_{N}\left(1+\lambda\left|z^{-1} \cdot x\right|\right)^{-N}
\end{aligned}
$$

for all $N \geq 0$.

\section{Proof of Theorem 3}

Since the operator norms of $T_{j}$ are equal to that of the rescaled operator $\widetilde{T}_{j}$ given by

$$
\widetilde{T}_{j} f(x, t)=2^{j \alpha} \int_{\mathbf{H}_{a}^{n}} \widetilde{K}_{j}\left((y, s)^{-1} \cdot(x, t)\right) f(y, s) d y d s
$$

where

$$
\widetilde{K}_{j}(x, t)=\vartheta(\rho(x, t)) \rho(x, t)^{-2 n-2-\alpha} e^{i 2^{j \beta} \rho(x, t)^{-\beta}}
$$

it suffices to establish estimate (3) for the rescaled operators $\widetilde{T}_{j}$.

Since the $\widetilde{T}_{j}$ are local operators, in the sense that the support of $\widetilde{T}_{j} f$ is always contained in a fixed dilate of some nonisotropic ball containing the support of $f$, we may make the additional assumption that the integral kernels above have compact support in both $(x, t)$ and $(y, s)$. Estimate $(3)$ for $\widetilde{T}_{j}$ then follows from Proposition 6 once we have verified the non-degeneracy condition (4) in this setting.

It is well known that

$$
X_{j}^{\ell}=\frac{\partial}{\partial x_{j}}+2 a x_{j+n} \frac{\partial}{\partial t}, \quad X_{j+n}^{\ell}=\frac{\partial}{\partial x_{j+n}}-2 a x_{j} \frac{\partial}{\partial t} \quad j=1, \ldots, n,
$$

and $T=\frac{\partial}{\partial t}$ form a real basis for the Lie algebra of left-invariant vector fields on $\mathbf{H}_{a}^{n}$, while

$$
X_{j}^{r}=\frac{\partial}{\partial x_{j}}-2 a x_{j+n} \frac{\partial}{\partial t}, \quad X_{j+n}^{r}=\frac{\partial}{\partial x_{j+n}}+2 a x_{j} \frac{\partial}{\partial t} \quad j=1, \ldots, n,
$$

and $T=\frac{\partial}{\partial t}$ form a real basis for the Lie algebra of right-invariant vector fields. 
For convenience we shall use synonymously $X_{2 n+1}^{\ell}=X_{2 n+1}^{r}=T$, and furthermore denote

We note that

$$
X^{\ell}=\left(X_{1}^{\ell}, \ldots, X_{2 n+1}^{\ell}\right) \quad \text { and } \quad X^{r}=\left(X_{1}^{r}, \ldots, X_{2 n+1}^{r}\right) .
$$

where $\widetilde{\varphi}(x)=\varphi\left((x, t)^{-1}\right)$, and hence

$$
-\left[X_{j}^{r} \widetilde{\varphi}\right](x, t)=\left[X_{j}^{\ell} \varphi\right]\left((x, t)^{-1}\right),
$$

$$
X_{j}^{\ell} Y_{k}^{\ell}\left[\varphi\left((y, s)^{-1} \cdot(x, t)\right)\right]=-\left[X_{j}^{\ell} X_{k}^{r} \varphi\right]\left((y, s)^{-1} \cdot(x, t)\right) .
$$

The non-degeneracy condition (4) in this setting is therefore equivalent to the following.

Proposition 7. Let $\Phi(x, t)=\rho(x, t)^{-\beta}$ with $\beta>0$. If $(x, t) \neq(0,0)$ and either

(i) $\rho(x, t)=\rho_{1}(x, t)$ with $0<a^{2}<C_{\beta}$, or

(ii) $\rho(x, t)=\rho_{2}(x, t)$ with $a^{2} \leq 1$,

then

$$
\operatorname{det}\left(X_{j}^{\ell} X_{k}^{r} \Phi(x, t)\right) \neq 0 .
$$

Theorem 3 now follows immediately for $b=1$, the proof in general follows from the observation that $\mathbf{H}_{a}^{n}$ is isomorphic to $\mathbf{H}_{a / b}^{n}$ with the explicit isomorphism being given by

$$
\phi(x, t)=(b x, b t) .
$$

\section{The determinant calculations}

The purpose of this section is to prove Proposition 7 , however we shall start by stating and sketching the proof of a related negative result for the quasi-norm $\rho_{3}(x, t)$ on $\mathbf{H}_{a}^{1}$. Outlining this argument first will be instructive as it is simpler than, while still similar to, those for Proposition 7.

Proposition 8. Let $n=1$ and $\Phi(x, t)=\rho_{3}(x, t)^{-\beta}$, then

$$
\operatorname{det}\left(X_{j}^{\ell} X_{k}^{r} \Phi(x, t)\right)=0
$$

along the lines $\left(0, x_{2}, 0\right)$ and $\left(x_{1}, 0,0\right)$.

Proof. Let $\varphi_{3}(x, t)=\rho_{3}(x, t)^{4}=x_{1}^{4}+x_{2}^{4}+t^{2}$. It is straightforward to see that the 'mixed' Hessian of $\Phi$ is given by

$$
X_{j}^{\ell} X_{k}^{r} \Phi(x, t)=-\frac{\beta}{4} \varphi_{3}^{-(\beta+8) / 4}\left\{\varphi_{3} X_{j}^{\ell} X_{k}^{r} \varphi_{3}-\frac{\beta+4}{4} X_{j}^{\ell} \varphi_{3} X_{k}^{r} \varphi_{3}\right\} .
$$

For convenience both here and in the proofs of both parts of Proposition 7 we define

$$
A:=X_{j}^{\ell} X_{k}^{r} \varphi_{3} \quad \text { and } \quad B:=X_{j}^{\ell} \varphi_{3} X_{k}^{r} \varphi_{3} .
$$

Now since $\operatorname{rank}(B)=1$ it follows that

$$
\begin{aligned}
& \operatorname{det}\left(\varphi_{3} A-\frac{\beta+4}{4} B\right) \\
& =\varphi_{3}^{2}\left\{\varphi_{3} \operatorname{det}(A)-\frac{\beta+4}{4}\left\{\operatorname{det}\left(\begin{array}{l}
\mathbf{b}_{1} \\
\mathbf{a}_{2} \\
\mathbf{a}_{3}
\end{array}\right)+\operatorname{det}\left(\begin{array}{l}
\mathbf{a}_{1} \\
\mathbf{b}_{2} \\
\mathbf{a}_{3}
\end{array}\right)+\operatorname{det}\left(\begin{array}{l}
\mathbf{a}_{1} \\
\mathbf{a}_{2} \\
\mathbf{b}_{3}
\end{array}\right)\right\}\right\} \text {, }
\end{aligned}
$$


where $\mathbf{a}_{j}=\left(a_{j 1}, a_{j 2}, a_{j 3}\right)$ and $\mathbf{b}_{j}=\left(b_{j 1}, b_{j 2}, b_{j 3}\right)$.

It is easy to verify that

$$
A=2(C+a D)
$$

where

$$
C=\left(\begin{array}{ccc}
6 x_{1}^{2} & 2 t & 0 \\
-2 t & 6 x_{2}^{2} & 0 \\
0 & 0 & 0
\end{array}\right) \quad \text { and } \quad D=\left(\begin{array}{ccc}
-4 a x_{2}^{2} & 4 a x_{1} x_{2} & 2 x_{2} \\
4 a x_{1} x_{2} & -4 a x_{1}^{2} & -2 x_{1} \\
-2 x_{2} & 2 x_{1} & 1 / a
\end{array}\right)
$$

Since $\operatorname{rank}(D)=1$ it follows that

$$
\operatorname{det}(A)=8 \operatorname{det}\left(\begin{array}{cc}
6 x_{1}^{2} & 2 t \\
-2 t & 6 x_{2}^{2}
\end{array}\right)=32\left(9 x_{1}^{2} x_{2}^{2}+t^{2}\right) .
$$

For the first of the remaining three determinants we note that

$$
\operatorname{det}\left(\begin{array}{l}
\mathbf{b}_{1} \\
\mathbf{a}_{2} \\
\mathbf{a}_{3}
\end{array}\right)=8 X_{1}^{\ell} \varphi_{3} \operatorname{det}(E+a F)
$$

where

$$
E=\left(\begin{array}{ccc}
2 x_{1}^{3} & 2 x_{2}^{3} & 0 \\
-2 t & 6 x_{2}^{2} & 0 \\
0 & 0 & 0
\end{array}\right) \quad \text { and } \quad F=\left(\begin{array}{ccc}
-2 x_{2} t & 2 x_{1} t & t / a \\
4 a x_{1} x_{2} & -4 a x_{1}^{2} & -2 x_{1} \\
-2 x_{2} & 2 x_{1} & 1 / a
\end{array}\right)
$$

Using the fact that $\operatorname{rank}(F)=1$ we then see that

$$
\operatorname{det}\left(\begin{array}{l}
\mathbf{b}_{1} \\
\mathbf{a}_{2} \\
\mathbf{a}_{3}
\end{array}\right)=8 X_{1}^{\ell} \varphi_{3} \operatorname{det}\left(\begin{array}{cc}
2 x_{1}^{3} & 2 x_{2}^{3} \\
-2 t & 6 x_{2}^{2}
\end{array}\right) .
$$

In an almost identical manner we can also obtain that

$$
\operatorname{det}\left(\begin{array}{l}
\mathbf{a}_{1} \\
\mathbf{b}_{2} \\
\mathbf{a}_{3}
\end{array}\right)=8 X_{2}^{\ell} \varphi_{3} \operatorname{det}\left(\begin{array}{cc}
6 x_{1}^{2} & 2 t \\
2 x_{1}^{3} & 2 x_{2}^{3}
\end{array}\right)
$$

and

$$
\begin{gathered}
\operatorname{det}\left(\begin{array}{l}
\mathbf{a}_{1} \\
\mathbf{a}_{2} \\
\mathbf{b}_{3}
\end{array}\right) \\
=8 X_{3}^{\ell} \varphi_{3}\left\{2 a x_{2} \operatorname{det}\left(\begin{array}{cc}
-2 t & 6 x_{2}^{2} \\
2 x_{1}^{3} & 2 x_{2}^{3}
\end{array}\right)+2 a x_{1} \operatorname{det}\left(\begin{array}{cc}
6 x_{1}^{2} & 2 t \\
2 x_{1}^{3} & 2 x_{2}^{3}
\end{array}\right)+t \operatorname{det}\left(\begin{array}{cc}
6 x_{1}^{2} & 2 t \\
-2 t & 6 x_{2}^{2}
\end{array}\right)\right\},
\end{gathered}
$$

we leave the details to the reader. Bringing all of this together we get that

$$
\begin{gathered}
\operatorname{det}\left(\varphi_{3} A-\frac{\beta+4}{4} B\right) \\
=-16 \varphi_{3}^{2}\left\{6(\beta+1) \varphi_{3} x_{1}^{2} x_{2}^{2}+(\beta+2) t^{4}+3(\beta+4) x_{1}^{2} x_{2}^{2} t^{2}-2\left(x_{1}^{4}+x_{2}^{4}\right) t^{2}\right\} .
\end{gathered}
$$


5.1. Proof of Proposition 7, part (i). Let $\varphi_{1}(x, t)=\rho_{1}(x, t)^{4}=|x|^{4}+t^{2}$. It is straightforward to see that the 'mixed' Hessian of $\Phi$ is given by

$$
X_{j}^{\ell} X_{k}^{r} \Phi(x, t)=-\frac{\beta}{4} \varphi_{1}^{-(\beta+8) / 4}\left\{\varphi_{1} X_{j}^{\ell} X_{k}^{r} \varphi_{1}-\frac{\beta+4}{4} X_{j}^{\ell} \varphi_{1} X_{k}^{r} \varphi_{1}\right\} .
$$

We again define $A:=X_{j}^{\ell} X_{k}^{r} \varphi_{1}$ and $B:=X_{j}^{\ell} \varphi_{1} X_{k}^{r} \varphi_{1}$. Since $\operatorname{rank}(B)=1$ it follows that

$$
\operatorname{det}\left(\varphi_{1} A-\frac{\beta+4}{4} B\right)=\varphi_{1}^{2 n}\left\{\varphi_{1} \operatorname{det}(A)-\frac{\beta+4}{4} \sum_{j=1}^{2 n+1} \operatorname{det}\left(\begin{array}{c}
\mathbf{a}_{1} \\
\vdots \\
\mathbf{b}_{j} \\
\vdots \\
\mathbf{a}_{2 n+1}
\end{array}\right)\right\},
$$

where $\mathbf{a}_{j}=\left(a_{j 1}, \ldots, a_{j 2 n+1}\right)$ and $\mathbf{b}_{j}=\left(b_{j 1}, \ldots, b_{j 2 n+1}\right)$.

It is an easy calculation to see that

$$
A=4\left(\begin{array}{cc}
C & 0 \\
0 & 0
\end{array}\right)+8\left(\begin{array}{cc}
D & 0 \\
0 & 0
\end{array}\right)+4 a E \text { and } B=4|x|^{2}\left(\begin{array}{ll}
F & 0
\end{array}\right)+4 a t G
$$

where

$$
\begin{gathered}
C=|x|^{2} I+a t J, \quad D=x x^{\mathrm{t}}, \quad E=\left(\begin{array}{cc}
2 a(J x)\left(x^{\mathrm{t}} J\right) & J x \\
x^{\mathrm{t}} J & 1 / 2 a
\end{array}\right), \\
F=\left(X^{\ell} \varphi_{1}\right) x^{\mathrm{t}}, \quad \text { and } \quad G=\left(\begin{array}{ll}
\left(X^{\ell} \varphi_{1}\right)\left(x^{\mathrm{t}} J\right) & X^{\ell} \varphi_{1} / 2 a
\end{array}\right)
\end{gathered}
$$

with $J$ the standard symplectic matrix on $\mathbf{R}^{2 n}$ coming from the group structure.

Since both $\operatorname{rank}(D)=1$ and $\operatorname{rank}(E)=1$ it follows that

$$
\begin{aligned}
\operatorname{det}(A) & =2^{4 n+1} \operatorname{det}(C+2 D) \\
& =2^{4 n+1}\left\{\left(|x|^{4}+a^{2} t^{2}\right)^{n}+\frac{1}{2}\left(|x|^{4}+a^{2} t^{2}\right)^{n-1} \sum_{j=1}^{2 n} x_{j} X_{j}^{\ell} \varphi_{1}\right\} \\
& =2^{4 n+1}\left(|x|^{4}+a^{2} t^{2}\right)^{n-1}\left(3|x|^{4}+a^{2} t^{2}\right) .
\end{aligned}
$$

Further careful calculations utilizing particular rank one matrices lead to the identities

$$
\begin{aligned}
\sum_{j=1}^{2 n} \operatorname{det}\left(\begin{array}{c}
\mathbf{a}_{1} \\
\vdots \\
\mathbf{b}_{j} \\
\vdots \\
\mathbf{a}_{2 n+1}
\end{array}\right) & =2^{4 n-1}\left(|x|^{4}+a^{2} t^{2}\right)^{n-1}|x|^{2} \sum_{j=1}^{2 n}\left(X_{j}^{\ell} \varphi_{1}\right)^{2} \\
& =2^{4 n+3}|x|^{4}\left(|x|^{4}+a^{2} t^{2}\right)^{n}
\end{aligned}
$$

and 


$$
\begin{aligned}
& \operatorname{det}\left(\begin{array}{c}
\mathbf{a}_{1} \\
\vdots \\
\mathbf{a}_{2 n} \\
\mathbf{b}_{2 n+1}
\end{array}\right)=4^{2 n+1} a X_{2 n+1}^{\ell} \varphi_{1} \operatorname{det}\left(\begin{array}{cc}
C+2 D & J x \\
|x|^{2} x^{\mathrm{t}} & t / 2 a
\end{array}\right) \\
& =4^{2 n+1} t\left(|x|^{4}+a^{2} t^{2}\right)^{n-1}\left\{\frac{1}{2} a|x|^{2} \sum_{j=1}^{n}\left(x_{j} X_{j+n}^{\ell} \varphi_{1}-x_{j+n} X_{j}^{\ell} \varphi_{1}\right)+t\left(3|x|^{4}+a^{2} t^{2}\right)\right\} \\
& =4^{2 n+1} t^{2}\left(|x|^{4}+a^{2} t^{2}\right)^{n-1}\left(\left(3-2 a^{2}\right)|x|^{4}+a^{2} t^{2}\right) .
\end{aligned}
$$

We omit the details, but observe that bringing this all together we obtain

$$
\sum_{j=1}^{2 n+1} \operatorname{det}\left(\begin{array}{c}
\mathbf{a}_{1} \\
\vdots \\
\mathbf{b}_{j} \\
\vdots \\
\mathbf{a}_{2 n+1}
\end{array}\right)=4^{2 n+1}\left(|x|^{4}+a^{2} t^{2}\right)^{n-1}\left\{2|x|^{8}+t^{2}\left(3|x|^{4}+a^{2} t^{2}\right)\right\}
$$

and consequently

$$
\operatorname{det}\left(\varphi_{1} A-\frac{\beta+4}{4} B\right)=-\left(4 \varphi_{1}\right)^{2 n}\left(|x|^{4}+a^{2} t^{2}\right)^{n-1} f_{1}(x, t)
$$

where

$$
f_{1}(x, t)=2(\beta+1)|x|^{8}+\left(3(\beta+2)-2 a^{2}\right)|x|^{4} t^{2}+(\beta+2) a^{2} t^{4} .
$$

By analyzing the discriminant

$$
\Delta=4 a^{4}-4(\beta+2)(2 \beta+5) a^{2}+9(\beta+2)^{2},
$$

we see that our Hessian will be non-degenerate provided

$$
a^{2}<C_{\beta}=\frac{\beta+2}{2}\left(2 \beta+5+\sqrt{(2 \beta+5)^{2}-9}\right) .
$$

Remark 9. We conclude by remarking that when $a^{2} \geq C_{\beta}$ the Hessian degenerates along the paraboloids

$$
|x|^{4}=\frac{2 a^{2}-3(\beta+2) \pm \sqrt{\Delta}}{4(\beta+1)} t^{2}
$$

5.2. Proof of Proposition 7, part (ii). We start by letting $\varphi_{2}(x, t)=\rho_{2}(x, t)^{2}$ and noting that as a consequence $\varphi_{2}$ must satisy the identity

$$
\varphi_{2}^{-1}|x|^{2}+\varphi_{2}^{-2} t^{2}=1 \text {. }
$$


Arguing as in the proof of Proposition 7, part (i) (and using the same notation) we see that $\varphi_{2}$ satisfies

$$
\operatorname{det}\left(\varphi_{2} A-\frac{\beta+2}{2} B\right)=\varphi_{2}^{2 n}\left\{\varphi_{2} \operatorname{det}(A)-\frac{\beta+2}{2} \sum_{j=1}^{2 n+1} \operatorname{det}\left(\begin{array}{c}
\mathbf{a}_{1} \\
\vdots \\
\mathbf{b}_{j} \\
\vdots \\
\mathbf{a}_{2 n+1}
\end{array}\right)\right\} .
$$

It follows from an easy, although somewhat lengthy, calculation that

$$
\mathcal{A}^{2} A=2 \mathcal{A} \varphi_{2}^{-2}\left(\begin{array}{ll}
C & 0 \\
0 & 0
\end{array}\right)+2 \mathcal{A} \varphi_{2}^{-4} D,
$$

where

$$
\begin{gathered}
C=\varphi_{2} I+2 a t J \\
D=\left(t X^{\ell} \varphi_{2}-\varphi_{2}(2 a J x, 1)\right)\left(t X^{r} \varphi_{2}-\varphi_{2}(-2 a J x, 1)\right)^{\mathrm{t}},
\end{gathered}
$$

and

$$
\mathcal{A}=\varphi_{2}^{-2}|x|^{2}+2 \varphi_{2}^{-3} t^{2}=\varphi_{2}^{-1}+\varphi_{2}^{-3} t^{2} .
$$

Since $\operatorname{rank}(D)=1$ and

$$
\mathcal{A}\left(t X_{2 n+1}^{\ell} \varphi_{2}-\varphi_{2}\right)=-\varphi_{2}^{-1}|x|^{2}
$$

it follows that

$$
\operatorname{det}\left(\mathcal{A}^{2} A\right)=2^{2 n+1} \mathcal{A}^{2 n-1} \varphi_{2}^{-(4 n+6)}\left(\varphi_{2}^{2}+4 a^{2} t^{2}\right)^{n}|x|^{4} .
$$

While observing that certain matrices have rank one it is possible to verify that

$$
\begin{aligned}
\mathcal{A}^{4 n+2} \sum_{j=1}^{2 n} \operatorname{det}\left(\begin{array}{c}
\mathbf{a}_{1} \\
\vdots \\
\mathbf{b}_{j} \\
\vdots \\
\mathbf{a}_{2 n+1}
\end{array}\right) & =4^{n} \mathcal{A}^{2 n-1} \varphi_{2}^{-(4 n+1)}\left(\varphi_{2}^{2}+4 a^{2} t^{2}\right)^{n-1}|x|^{2} \sum_{j=1}^{2 n}\left(\mathcal{A} X_{j}^{\ell} \varphi_{2}\right)^{2} \\
& =4^{n+1} \mathcal{A}^{2 n-1} \varphi_{2}^{-(4 n+5)}\left(\varphi_{2}^{2}+4 a^{2} t^{2}\right)^{n}|x|^{4}
\end{aligned}
$$

and

$$
\mathcal{A}^{4 n+2} \operatorname{det}\left(\begin{array}{c}
\mathbf{a}_{1} \\
\vdots \\
\mathbf{a}_{2 n} \\
\mathbf{b}_{2 n+1}
\end{array}\right)=4^{n} \mathcal{A}^{2 n+2} \varphi_{2}^{-4 n} X_{2 n+1}^{\ell} \varphi_{2} X_{2 n+1}^{r} \varphi_{2} \operatorname{det}(C)+\sum_{j=1}^{2 n} \operatorname{det}\left(\begin{array}{c}
\mathbf{c}_{1} \\
\vdots \\
\mathbf{d}_{j} \\
\vdots \\
\mathbf{c}_{2 n} \\
\mathbf{b}_{2 n+1}
\end{array}\right) .
$$

It then follows from a careful calculation and the identity

$$
\mathcal{A} \sum_{j=1}^{n}\left\{x_{j} X_{j+n}^{\ell} \varphi_{2}-x_{j+n} X_{j}^{\ell} \varphi_{2}\right\}=-8 a^{2} \varphi_{2}^{-1} t|x|^{2}
$$


that

$$
\begin{gathered}
\sum_{j=1}^{2 n} \operatorname{det}\left(\begin{array}{c}
\mathbf{c}_{1} \\
\vdots \\
\mathbf{d}_{j} \\
\vdots \\
\mathbf{c}_{2 n} \\
\mathbf{b}_{2 n+1}
\end{array}\right) \\
=4^{n+1} \mathcal{A}^{2 n} \varphi_{2}^{-(4 n+4)}\left(\varphi_{2}^{2}+4 a^{2} t^{2}\right)^{n-1} X_{2 n+1}^{\ell} \varphi_{2}\left\{\left(\varphi_{2}^{2}+4 a^{2} t^{2}\right)-2 \mathcal{A} a^{2} \varphi_{2}^{3}\right\}|x|^{2} t .
\end{gathered}
$$

Bringing this all together we see that

$$
\begin{gathered}
\mathcal{A}^{4 n+2} \sum_{j=1}^{2 n+1} \operatorname{det}\left(\begin{array}{c}
\mathbf{a}_{1} \\
\vdots \\
\mathbf{b}_{j} \\
\vdots \\
\mathbf{a}_{2 n+1}
\end{array}\right) \\
=4^{n+1} \mathcal{A}^{2 n} \varphi_{2}^{-(4 n+4)}\left(\varphi_{2}^{2}+4 a^{2} t^{2}\right)^{n-1}\left\{\left(\varphi_{2}^{2}+4 a^{2} t^{2}\right) t^{2}+\varphi_{2}^{3}|x|^{2}\right\}
\end{gathered}
$$

and consequently

$$
\mathcal{A}^{4 n+2} \operatorname{det}\left(\varphi_{2} A-\frac{\beta+2}{2} B\right)=-2^{2 n+1} \mathcal{A}^{2 n-1} \varphi_{2}^{-(2 n+5)}\left(\varphi_{2}^{2}+4 a^{2} t^{2}\right)^{n-1} f_{2}(x, t)
$$

where

$$
f_{2}(x, t)=\mathcal{A} \varphi_{2}^{4}|x|^{2}+4 \varphi_{2}^{2} t^{2}\left(1-a^{2}\right)+16 a^{2} t^{4}+4 a^{2} \varphi_{2}^{-2} t^{6} .
$$

\section{Further comparisons}

6.1. Nonisotropic $\mathbf{R}^{2 n+1}$. When $a=0$ (not a Heisenberg type group, but still a homogeneous group) we of course have $X_{j}^{\ell}=X_{j}^{r}=\partial / \partial x_{j}$ and it is then straightforward to verify that in this case we have the following:

If $\rho(x, t)=\rho_{1}(x, t)$, then (in the notation of Section 5) we get that

$$
\operatorname{det}\left(\varphi_{1} A-\frac{\beta+4}{4} B\right)=-b\left(4 \varphi_{1}\right)^{2 n}|x|^{4 n}\left\{2(\beta+1)|x|^{4}+3(\beta+2) t^{2}\right\}
$$

and in particular the Hessian degenerates along the line $(0, t)$.

While if $\rho(x, t)=\rho_{2}(x, t)$, then

$$
\mathcal{A}^{4 n+2} \operatorname{det}\left(\varphi_{2} A-\frac{\beta+2}{2} B\right)=-2^{2 n+1} \mathcal{A}^{2 n-1} \varphi_{2}^{-5}\left\{\beta \mathcal{A} \varphi_{2}^{3}+\mathcal{A} \varphi_{2}^{2}|x|^{2}+4 t^{2}\right\}
$$

which is clearly non-degenerate.

Remark 10. Sharp $L^{p}$ estimates for nonisotropic operators of this type in the plane $\mathbf{R}^{1+1}$ equipped with the dilation structure $\delta \circ(x, t)=\left(\delta^{a_{1}} x, \delta^{a_{2}} t\right)$ were obtained by Shayya [8] using Fourier transform methods. In particular, Shayya showed that if you take $\rho=\rho_{2}$ to be the nonisotropic Minkowski functional associated to the unit ball in $\mathbf{R}^{2}$, then

$$
\left|\widehat{K_{\alpha, \beta}}(\xi, \tau)\right| \leq C\left(1+\max \left\{|\xi|^{1 /\left(a_{1}+\beta\right)},|\tau|^{1 /\left(a_{2}+\beta\right)}\right\}\right)^{\alpha-\beta} .
$$


We take this opportunity to point out that in this setting it is rather straightforward to observe that equivalent norms can give rise to different $L^{2}$ behavior. To be more precise, if we instead set $\rho=\rho_{3}$ where $\rho_{3}(x, t)=\left(x^{2 a_{2}}+t^{2 a_{1}}\right)^{1 / 2 a_{1} a_{2}}$ with $a_{2} \geq a_{1}$, then by applying the method of stationary phase one can see that in order for the inequality

$$
\left|\widehat{K_{\alpha, \beta}}(\xi, \tau)\right| \leq C
$$

to hold it is necessary that $\alpha \leq\left(a_{2}+1\right) \beta / 2 a_{2}$.

6.2. Polarized Heisenberg group. The polarized Heisenberg group $\mathbf{H}_{a, \mathrm{pol}}^{n}$ is isomorphic to the full Heisenberg group $\mathbf{H}_{a}^{n}$ and has the multiplication law

$$
(x, t) \cdot(y, s)=\left(x+y, s+t-2 a x^{\mathrm{t}} J_{\text {pol }} y\right)
$$

where again $a$ is a nonzero real number, but now $J_{\text {pol }}$ denotes the matrix on $\mathbf{R}^{2 n}$,

$$
J_{\mathrm{pol}}=\left(\begin{array}{cc}
0 & I_{n} \\
0 & 0
\end{array}\right)
$$

In particular, if $n=1$ and $a=-1 / 2$, then this is the $m=3$ case of the groups of $m \times m$ upper-triangular matrices with ones along the diagonal; see Stein [9].

When $n=1$ the corresponding Lie algebra is generated by the left-invariant vector fields

$$
X_{1}^{\ell}=\frac{\partial}{\partial x_{1}}, \quad X_{2}^{\ell}=\frac{\partial}{\partial x_{2}}+2 a x_{1} \frac{\partial}{\partial t}, \quad \text { and } X_{3}^{\ell}=\frac{\partial}{\partial t},
$$

with the right-invariant vector fields being given by

$$
X_{1}^{r}=\frac{\partial}{\partial x_{1}}+2 a x_{1} \frac{\partial}{\partial t}, \quad X_{2}^{r}=\frac{\partial}{\partial x_{2}}, \quad \text { and } X_{3}^{r}=\frac{\partial}{\partial t} .
$$

Proposition 11. Let $n=1$ and $\Phi(x, t)=\rho_{1}(x, t)^{-\beta}$, then for all real $a$

$$
\operatorname{det}\left(X_{j}^{\ell} X_{k}^{r} \Phi(x, t)\right)=0
$$

along the line $(0, t)$.

In actual fact, using again the notation of the previous section, we have that

$$
\operatorname{det}\left(\varphi_{1} A-\frac{\beta+4}{4} B\right)=-16\left\{2(\beta+1)|x|^{8}+3(\beta+2)|x|^{4} t^{2}-2(\beta+2) a \varphi_{1} x_{1} x_{2} t\right\} .
$$

We leave the details to the reader.

Proposition 12. Let $n=1$ and $\Phi(x, t)=\rho_{2}(x, t)^{-\beta}$ with $\beta>0$, then for $(x, t) \neq$ $(0,0)$

$$
\operatorname{det}\left(X_{j}^{\ell} X_{k}^{r} \Phi(x, t)\right) \neq 0
$$

provided $a^{2} \leq 1$

Proof. A calculation similar to those above yields

$$
\begin{aligned}
& \mathcal{A}^{6} \operatorname{det}\left(\varphi_{2} A-\frac{\beta+2}{2} B\right) \\
& =-8 \mathcal{A} \varphi_{2}^{-7}\left\{\beta \mathcal{A} \varphi_{2}\left(\varphi_{2}^{2}-a x_{1} x_{2} t\right)+\left(2 \mathcal{A} \varphi_{2}^{3}-2 \mathcal{A} \varphi_{2} a x_{1} x_{2} t-\left(x_{1}^{2}+x_{2}^{2}\right)^{2}\right)\right\} .
\end{aligned}
$$


It is then easy to see that

$$
\begin{aligned}
2 \mathcal{A} \varphi_{2}^{3}-2 \mathcal{A} \varphi_{2} a x_{1} x_{2} t-\left(x_{1}^{2}+x_{2}^{2}\right)^{2} & \geq \mathcal{A} \varphi_{2}\left(\varphi_{2}\left(x_{1}^{2}+x_{2}^{2}\right)-2 a x_{1} x_{2} t\right) \\
& \geq \mathcal{A} \varphi_{2}\left(x_{1}^{2}+x_{2}^{2}\right)\left(\varphi_{2}-|a t|\right),
\end{aligned}
$$

which is clearly nonnegative if $a^{2} \leq 1$, since it follows from (7) that $\varphi_{2} \geq|t|$. In the last line above we used the easily verifiable inequality

$$
2 a x_{1} x_{2} t \leq\left(x_{1}^{2}+x_{2}^{2}\right)|a t| .
$$

From this inequality it also follows that

$$
\varphi_{2}^{2}-2 a x_{1} x_{2} t \geq 2\left|x_{1} x_{2}\right|\left(\varphi_{2}-|a t|\right)
$$

and hence, if we again assume that $a^{2} \leq 1$, we see that

$$
\varphi_{2}^{2}-a x_{1} x_{2} t \geq \varphi_{2}^{2} / 2 \text {. }
$$

\section{References}

[1] A. Erdélyi, Asymptotic forms for Laguerre polynomials, J. Indian Math. Soc. (N.S.) 241960 , 235-250 (1961).

[2] C. Fefferman, Inequalities for strongly singular convolution operators, Acta Math. 124 (1970), $9-36$.

[3] C. Fefferman and E. M. Stein, $H^{p}$ spaces of several variables, Acta Math. 129 (1972), no. 3-4, 137-193.

[4] I. I. Hirschman, Multiplier Transforms I, Duke Math. J. 26 (1956), 222-242.

[5] L. Hörmander, Oscillatory integrals and multipliers on $F L^{p}$, Ark. Math. 11 (1973), 1-11.

[6] N. Lyall, A class of strongly singular Radon transforms on the Heisenberg group, Proc. Edinb. Math. Soc. 50 (2007), 429-457.

[7] _ Strongly Singular Convolution Operators on the Heisenberg group, Trans. Amer. Math. Soc. 359 (2007), 4467-4488.

[8] B. Shayya, Nonisotropic strongly singular integral operators, Trans. Amer. Math. Soc., 354, No. 12 (2002), pp. 4893-4907.

[9] E. M. Stein, Harmonic Analysis: Real-Variable Methods, Orthogonality, and Oscillatory Integrals, Princeton Univ. Press, Princeton, 1993.

[10] S. Wainger, Special Trigonometric Series in k Dimensions, Memoirs of the AMS 59, American Math. Soc., 1965.

School of Mathematics, The University of Edinburgh, JCM Bullding, The King's Buildings, Edinburgh EH9 3JZ, United Kingdom

E-mail address: N.Laghi@ed.ac.uk

Department of Mathematics, The University of Georgia, Boyd Graduate Studies ReSearch Center, Athens, GA 30602, USA

E-mail address: lyall@math.uga.edu 